\title{
Positive association between artificially sweetened beverage consumption and incidence of diabetes
}

\author{
Allison C. Sylvetsky Meni ${ }^{1,2} \cdot$ Susan E. Swithers $^{3}$ • Kristina I. Rother ${ }^{1}$
}

Received: 15 May 2015 / Accepted: 30 June 2015 / Published online: 18 July 2015

(C) Springer-Verlag (outside the USA) 2015

Keywords Artificial sweeteners · Artificially sweetened beverages $\cdot$ Diabetes $\cdot$ Diet soda $\cdot$ Non-nutritive sweeteners . Obesity $\cdot$ Soda $\cdot$ Sugar-sweetened beverages

\section{Abbreviations \\ ASB Artificially sweetened beverages \\ SSB Sugar-sweetened beverages}

To the Editor: O'Connor and colleagues are to be applauded for evaluating the effects of sweetened and unsweetened nonalcoholic beverage intake on the incidence of type 2 diabetes in 24,653 individuals [1]. In addition to the large cohort size, the study participants were followed for almost 11 years. The finding that intake of sugar-sweetened beverages (SSB, soda and milk) was strongly associated with the eventual development of type 2 diabetes is very important and not unexpected. However, we believe that another finding is particularly noteworthy, namely, intake of artificially sweetened beverages (ASB) was an equally strong predictor of type 2 diabetes.

Kristina I. Rother

kristina.rother@nih.gov

1 Section on Pediatric Diabetes and Metabolism, National Institute of Diabetes and Digestive and Kidney Diseases, National Institutes of Health, 9000 Rockville Pike, Building 10, Room 8C432A, Bethesda, MD 20892-1645, USA

2 Department of Exercise and Nutrition Sciences, Milken Institute School of Public Health, The George Washington University, Washington, DC, USA

3 Department of Psychological Sciences, Purdue University, West Lafayette, IN, USA
When corrected for BMI and waist circumference, the significant association between sugar-sweetened soft drinks and type 2 diabetes persisted whereas the relationship between ASB intake and type 2 diabetes was attenuated, pointing to obesity as an important link between ASB and type 2 diabetes. In fact, the prevalence of obesity was strikingly higher in the ASB cohort than among the SSB consumers, despite the fact that their energy intakes were similar (ASB consumers: BMI $27.2 \pm 4.2 \mathrm{~kg} / \mathrm{m}^{2}$, obesity prevalence $20.3 \%$, energy intake $7,899 \pm 2,046 \mathrm{~kJ} /$ day; SSB consumers: BMI $26.2 \pm 3.9 \mathrm{~kg} / \mathrm{m}^{2}$, obesity prevalence $14.4 \%$, energy intake $8,389 \pm 2,071 \mathrm{~kJ} /$ day). The authors state that the positive association between ASB intake and type 2 diabetes may be an artefact of reverse causality, meaning that heavier individuals consume more beverages containing artificial sweeteners to prevent additional weight gain or even promote weight loss. In our opinion, a direct detrimental effect of artificial sweeteners on metabolic health warrants further consideration, especially in light of recent findings in animal [2, 3] and human [2, 4, 5] studies. These findings include interference of artificial sweeteners with learned signals linking sweet taste to its post-ingestive consequences [3] and induction of changes in the gut microbiome [2]. Both may impair blood glucose regulation and enhance metabolic efficiency. In addition, stimulation of insulin secretion by artificial sweeteners, as observed in in vitro studies [6] and in a recent human study evaluating acute ASB consumption [4], offers another plausible mechanism that could explain the relationship between ASB and greater adiposity.

Based on mathematical modelling, O'Connor and colleagues [1] predict that replacing SSB with ASB would not reduce the diabetes risk. This supports the notion that even if artificial sweeteners reduce overall energy intake, they may exert adverse metabolic effects, especially by increasing weight and adiposity. These findings have important implications for individuals of all ages, including children, whose 
schools increasingly replace SSB with diet and low-energy beverages in their vending machines [7].

It should also be noted that if ASB contribute to weight gain and increased adiposity, statistical adjustments for these factors in studies of the effects of ASB on disease risk may be equivalent to 'throwing the baby out with the bathwater'. Evidence from prospective cohort studies has indicated that ASB consumption may directly contribute to significant increases in waist circumference independently of changes in BMI [5], as well as to significant increases in BMI [8]. As a result, O'Connor's data add to a growing number of studies which document that ASB consumption fails to mitigate the risk of obesity-related negative health outcomes and may instead increase their likelihood.

Acknowledgement This work was supported in part by the intramural research program of the National Institute of Diabetes, Digestive and Kidney Diseases.

Duality of interest The authors declare that there is no duality of interest associated with this manuscript.

Contribution statement All authors were responsible for drafting the article and revising it critically for important intellectual content. All authors approved the version to be published.

\section{References}

1. O'Connor L, Imamura F, Lentjes M, Khaw K, Wareham N, Forouhi N (2015) Prospective associations and population impact of sweet beverage intake and type 2 diabetes, and effects of substitutions with alternative beverages. Diabetologia 58:1474-1483

2. Suez J, Korem T, Zeevi D, Zilberman-Schapira G et al (2014) Artificial sweeteners induce glucose intolerance by altering the gut microbiota. Nature 514:181-186

3. Swithers SE, Laboy AF, Clark K, Cooper S, Davidson TL (2012) Experience with the high-intensity sweetener saccharin impairs glucose homeostasis and GLP-1 release in rats. Behav Brain Res 233:1-14

4. Pepino MY, Tiemann CD, Patterson BW, Wice BM, Klein S (2013) Sucralose affects glycemic and hormonal responses to an oral glucose load. Diabetes Care 36:2530-2535

5. Fowler SP, Williams K, Hazuda HP (2015) Diet soda intake is associated with long-term increases in waist circumference in a biethnic cohort of older adults: The San Antonio Longitudinal Study of Aging. J Am Geriatr Soc 63:708-715

6. Nakagawa Y, Nagasawa M, Yamada S et al (2009) Sweet taste receptor expressed in pancreatic beta-cells activates the calcium and cyclic AMP signaling systems and stimulates insulin secretion. PLoS One 4: e5106

7. Sylvetsky A, Rother KI, Brown R (2011) Artificial sweetener use among children: epidemiology, recommendations, metabolic outcomes, and future directions. Pediatr Clin N Am 58:1467-1480

8. Fowler SP, Williams K, Resendez RG, Hunt KJ, Hazuda HP, Stern MP (2008) Fueling the obesity epidemic? Artificially sweetened beverage use and long-term weight gain. Obesity (Silver Spring) 16:1894-1900 